\title{
Modelo matemático para el control de costos de empresas productivas
}

\author{
Mathematical model for cost control of productive companies
}

María Pamela Solares Gantier

Artículo recibido en diciembre 2020

pame.solaresgantier@gmail.com

Arbitrado en febrero 2021

Aceptado en febrero 2021

Publicado en mayo 2021

ORCID: 0000-0002-2808-3341

Universidad San Francisco Xavier de Chuquisaca

Sucre - Bolivia

RESUMEN Uno de los problemas que atraviesan las empresas y organizaciones productivas de Bolivia es el control de costos, en especial los costos indirectos que son los más difíciles de administrar. El objetivo de esta investigación fue desarrollar un modelo matemático que permita identificar los costos que reducen las utilidades.

La metodología utilizada identificó las causas, como los costos directos e indirectos para llegar al efecto, representado como Costo Unitario Total, resultando un modelo multivariable y multifactorial.

Los resultados de la simulación muestran en la información final productos con alta y baja utilidad $y$, en algunos casos, con valores negativos, validando el modelo. Existen beneficios materiales y cualitativos que resultan de la aplicación del Modelo, por lo que se considera recomendable ejercitar su implantación en toda empresa que pretenda obtener mayores utilidades con la mejor utilización de sus recursos humanos y materiales.

Palabras clave: Costos Indirectos; Modelo Matemático; Rentabilidad; Control de costos; Costo unitario

ABSTRACT

Keywords:

Bolivian companies and organizations tend to have cost control problems, especially indirect ones that are the most difficult to manage, so the objective of this research, was to develop a mathematical model that allows identifying the costs that reduce profits.

The methodology used identified the causes as the direct and indirect costs to arrive at the effect represented as Total Unit Cost, resulting in a multivariate and multifactorial model.

The results of the simulation show in the final information products with high and low utility and, in some cases, with negative values, validating the model. There are material and qualitative benefits that result from the application of the Model, so it is considered advisable to exercise its implementation in any company that intends to obtain higher profits with the best use of its human and material resources.

Indirect Costs; Mathematical model; Cost effectiveness; Cost control; Unit cost 


\section{INTRODUCCIÓN}

La contabilidad financiera registra el estado de las cuentas empresariales y ayuda a determinar el costo total de producción con las cuentas de egresos. Si la producción, refiere muchos productos, los resultados no dicen mucho sobre cómo controlar los costos (Neuner \& Deakin, 2005). La contabilidad de costos es la puerta que permite ampliar los alcances limitados de la contabilidad financiera, en una empresa o industria de servicios o bienes, de manera que la alta dirección empresarial pueda tomar acciones relativas a los costos de producir o vender un producto cualquiera (Hansen et al., 2007).

La contabilidad de costos es un tema multifactorial y multivariable de dimensiones mayores y depende de: tamaño de la empresa, espectro de productos que presenta al mercado, complejidad de la producción y temperamento empresarial. Por supuesto, influye también si la empresa está dispuesta a pagar un manejo controlado de costos, adecuado a sus necesidades particulares, porque la mayoría de las veces no lo hacen. Si bien los conceptos son estándar, bajo una perspectiva actual la forma de realizar el control es como comprar un traje a medida (Sánchez, 2009).

Antiguamente el análisis de costos centraba su atención en dos elementos importantes, a saber: materia prima y mano de obra y, dejaba de lado los costos indirectos, que es el tercer elemento importante (Montoya et al., 2010). Al respecto, la basta bibliografía que antecedió a la automatización de proceso de datos por computador programable, deja clara la enorme dificultad de repartir algo que es de todos (para este análisis: los costos indirectos), entre varios, cuando no se tiene ninguna guía para hacerlo (William, 2010).
La contabilidad de costos es difícil de practicar y requiere más esfuerzo que la contabilidad financiera, pero ambas son indispensables y debieran llevarse paralelamente (Christopher, 2010). En los medios que tienen muchas limitaciones económicas, este control es casi un mito, como lo es el de control presupuestario. Por este motivo, es que hay empresas que a veces, sin percibirlo, soportan pérdidas que no se hacen visibles hasta que los síntomas se intensifican, hasta bordear la quiebra. Los síntomas pueden permanecer relativamente ocultos hasta que la diferencia entre la utilidad de gestión y la utilidad estimada con el sistema de costos, se aprecian y evidencian muy grandes. La principal causa es la falta de un sistema de control de costos a medida.

En respuesta a esta necesidad, en lo referente a la investigación, el objetivo que se buscó alcanzar fue desarrollar un modelo matemático que permita identificar los costos que reducen las utilidades en un proceso productivo. Se presenta a la conclusión un modelo multivariable que requiere para su operación, un sistema informático y una estructura de datos de avanzada para el control de costos.

La contribución práctica de este estudio es la que más justifica el esfuerzo y se la puede observar a corto plazo, tan pronto como se den los primeros cambios en la rentabilidad al cierre del ejercicio económico. El modelo provee, de manera ordenada, la información para una correcta toma de decisiones.

Debe resaltarse que el entorno multifactorial en el que se desarrollan las estimas usa indicadores originales para el prorrateo de los costos indirectos, estos tienen la cualidad de aportar información intermedia 
adicional. Si bien ilumina más el campo en que se realiza el análisis y se toman las decisiones, aumenta la complejidad de la base de datos por lo que es indispensable contar con el soporte de un sistema informático (Castán et al., 2003).

Existe suficiente teoría para entender el problema de costos y la forma de realizar un seguimiento contable, pero las contribuciones publicadas para administrar los costos indirectos son muy limitadas en lo referente a modelos y procesos que permiten acercarse sistemáticamente (algoritmo) a lo que se requiere: una acertada toma de decisiones. Asimismo, como los costos indirectos sólo pueden estimarse y definitivamente no calcularse (Rincón-Soto et al., 2019), hay muy pocos esfuerzos que hayan concluido en aportes que den luz suficiente, sobre la forma de controlarlos eficiente $y$, sobre todo, eficazmente.

\section{METODOLOGÍA}

Se trata de un estudio de corte transversal que fue realizado con un enfoque cuantitativo, puesto que el modelo que se propone expone la relación entre variables cuantificables y medibles numéricamente. El método teórico utilizado en la investigación fue el analíticosintético, mismo que se aplicó en el estudio del problema, partiendo del efecto hacia las causas, para analizarlas de manera individual (Hernández et al., 2010). Luego, fue aplicado en la síntesis del modelo, partiendo de las causas, en este caso los costos directos e indirectos, para estudiarlos en su totalidad como costo unitario total. La figura 1 ilustra la correlación de variables que corresponden al modelo antes mencionado.

\section{Figura 1}

Lógica de desarrollo y aplicación del Modelo

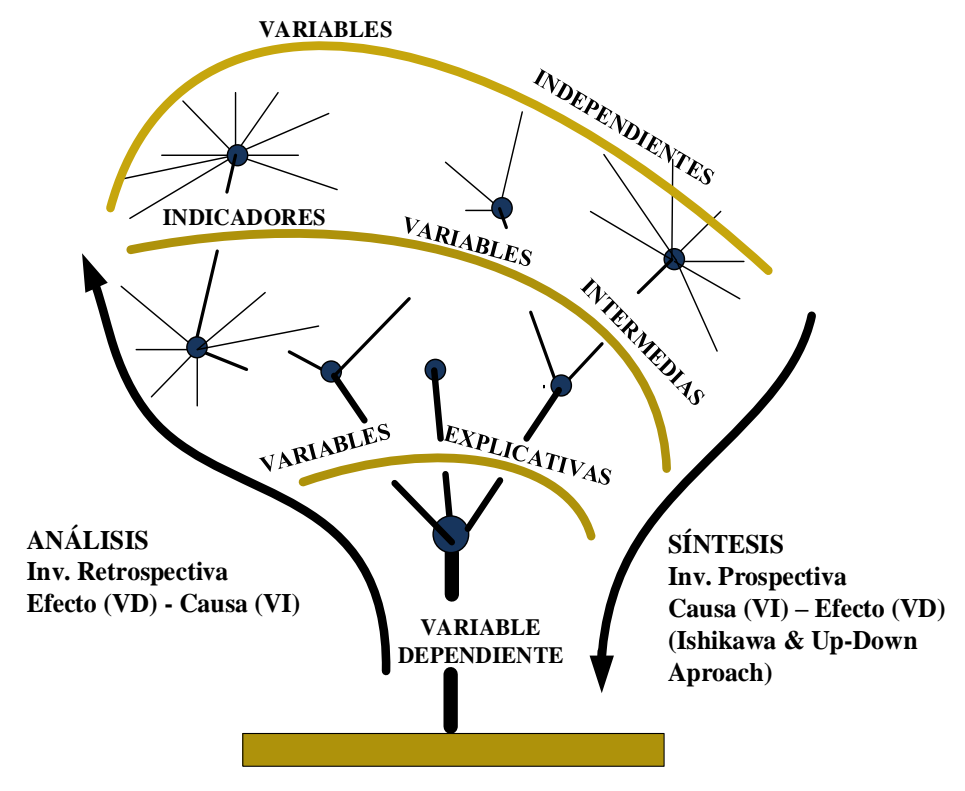


En la Figura 1 se identifican las variables independientes (Causas) como los costos directos e indirectos para llegar al "Efecto" representado por la variable dependiente que representa el "Costo Unitario Total".

Una vez consolidado el modelo, fue aplicado de forma práctica y validado, dando guías sobre los pasos sistemáticos a seguir, dejando expuesta la incidencia de los costos directos e indirectos, en los resultados de la gestión económica.

\section{RESULTADOS}

Cuando la empresa crece al punto que maneja una amplia gama de productos en un mercado de gran tamaño, o mejor aún, cuando se tiende a la producción masiva cerca de los rendimientos marginales decrecientes, la complejidad que conlleva el control de costos indirectos debe necesariamente apoyarse en un modelo matemático y un eficiente sistema informático.

Para lograr el objetivo, se planteó el algoritmo en términos de un modelo cuya complejidad depende de los niveles y detalle con los que se eligen las variables para llegar al resultado. Como se puede intuir, el modelo resultante es multivariable y multifactorial porque considera varios costos como variables; y varios coeficientes, para realizar el prorrateo de costos indirectos.

En virtud de lo expuesto, el desarrollo del modelo tiene como tareas: 1) Establecer una correlación de variables que permita determinar la composición de los diferentes costos; 2) Determinar las bases de afectación, factores y coeficientes de prorrateo que permitan la estima de los costos indirectos; 3 ) Sintetizar el modelo general de costos; 4) Estimar los costos directos e indirectos que intervienen en un proceso productivo (Aplicación).

También queda claro que la información sobre costos directos es laboriosa de obtener, pero no imposible, cosa que no sucede con los costos indirectos que permanecen ocultos o no identificados en todos los casos. Aquí se hace evidente la contribución práctica y el principal aporte de este trabajo.

\section{CORRELACIÓN DE VARIABLES $Y$ COMPOSICIÓN DE LOS COSTOS}

Se parte con base en dos generalizaciones: (1) En este trabajo, se manejan los costos esencialmente por lotes, debido a que la programación y el proceso productivo es por lote, así como la previsión de espacio de almacenamiento previo a la salida de la empresa o la fábrica. Por tanto, se utilizó el lote de producto promedio para facilitar el manipuleo informático; (2) Como la producción se realiza para una cantidad determinada de producto, el costo unitario se obtendrá dividiendo el costo del proceso por esta cantidad que refiere un lote de producto.

Se define entonces el costo total con sus dos grandes componentes: costo directo y costo indirecto. La estructura de costos puede variar ligeramente de una empresa a otra y es posible que se experimente que, algunos costos que resultan indirectos en un caso se comporten como costos directos en otro, cuando las variables se hagan fácilmente verificables y medibles.

Este modelo plantea como elementos obligatorios a considerar y cuantificar dentro de los costos directos: a) materia prima y partes, b) personal y mano de obra, c) uso de instalaciones, d) equipo y/o maquinaria (financiero y depreciación), e) almacenamiento 
de producto en preventa $y, f$ ) costo por insumos. Una vez se tiene identificados los componentes del costo directo se los detalla. El hecho de que algunos de estos no sean aplicables a un caso particular, no invalida el modelo, pues es solamente indicativo de que este componente debe ser simplemente desestimado.

Enfocando el análisis en los costos indirectos, se identifican cuatro componentes que influyen considerablemente en los resultados de estas estimas y son los relacionados con: a) salarios (SAL), b) operaciones y servicios generales (OyS), c) financiamiento y depreciación de activos (ACT, FD) $y, d$ ) estacionamiento de producto (EST), incluye almacenes, agencias, puntos de venta, etc.

Por la naturaleza de los gastos, pueden convenientemente agruparse estos componentes en dos: por un lado los salarios, operaciones y servicios generales y la depreciación y financiamiento de los activos, denominándolos $\mathrm{C}_{\mathrm{SOSA}, \mathrm{Ti}} \mathrm{y}$, por otra parte, está el costo de estacionamiento correspondiente al financiamiento de saldos en almacenes $y$ saldos deudores de clientes en agencias $y$ puntos de venta, este se lo denomina $C_{E S T, I}$.

\section{BASES DE AFECTACIÓN, FACTORES Y COEFICIENTES DE PRORRATEO}

Una base de afectación es la cantidad monetaria de un componente o rubro que sirve de base para el cálculo de un costo, entendida como la cantidad sobre la que actúa un coeficiente de prorrateo. En cada caso es necesario conceptualizar y establecer la base de afectación para su registro en la base de datos. Cada una es interpretada y modelada, para que la estima de costos indirectos se haga viable.

Se definen las bases de afectación para los costos directos a $\Delta_{P M O}, \Delta_{E M, F}, \Delta_{E M, D}$, como las diferencias entre el total registrado en estados o planillas y la suma de los i-ésimos costos directos.

En el caso de las bases de afectación para los componentes indirectos se tiene: a) La base afectable por carga salarial $\left(B A_{, S A L}\right)$, que corresponde a los salarios clasificados, administración, comercialización, programación, operación y mantenimiento y producción; b) Base afectable por gastos de operación y servicios $\left(B A_{, S A L}\right)$, que corresponde a los gastos varios; c) Base afectable por cargas de financiamiento $y$ depreciación de activos $\left(B A_{, O y S}\right)$, que se calcula tomando el costo financiero anual de activos y la depreciación; d) Base afectable por cargas por estacionamiento de producto $(B A, A C T)$, se calcula multiplicando los Saldos totales monetarios de almacenes, agencias y puntos de venta, promediados en el año, por la tasa de operación.

Estas bases de afectación servirán también como información para la determinación de coeficientes de prorrateo de manera que se obtenga información sobre la incidencia de: personal, mano de obra, equipo y maquinaria, en los costos indirectos y totales.

Una vez se han definido las bases de afectación para costos indirectos, se pone atención en la concepción de factores de ponderación y coeficientes de prorrateo.

El valor del coeficiente refleja la parte de la unidad que deberá atribuirse, de esa diferencia, a un determinado producto. A continuación, se detallan los principales coeficientes obtenidos 


\section{Coeficiente de prorrateo para personal y mano de obra}

En cuanto al coeficiente de prorrateo para personal y mano de obra $\left(\pi_{P M O}\right)$, la experiencia adquirida por la práctica cotidiana muestra que la diferencia $\Delta P M O$ es considerable, siendo necesario prorratearla con el coeficiente $\pi_{P M O, i}$. El valor de este coeficiente refleja la parte de la unidad que deberá atribuirse, de esa diferencia, a un determinado producto. En otras palabras, si el costo directo estimado por personal y mano de obra es una parte muy grande de los costos directos totales, entonces, en esa proporción le serán asignados los indirectos.

\section{Coeficientes de prorrateo para instalaciones técnicas, equipo $y$ maquinaria}

Por su parte, los coeficientes de prorrateo para instalaciones técnicas, equipo $y$ maquinaria $\left(\pi_{I E M}\right)$, incluyen dos tipos de costos, uno por la carga financiera sobre el capital actualizado que se tiene invertido trabajando $y$, otro, por la depreciación. Para cada caso, se define un Coeficiente-Prorrata que permite definir el costo total financiero o de depreciación, según corresponde, $\pi_{I E M, F, i}$ y $\pi_{I E M, D, i}$. Conceptualmente, estos coeficientes llevan la parte, que tiene un costo individual directo, del costo indirecto globalizado, que es conocido en los estados de revalorización, depreciación etc. En otras palabras, si el costo directo estimado es una parte muy grande del costo directo total por este concepto, entonces, en esa proporción le serán asignados los indirectos.

\section{Factor y coeficiente de la relación de costos directos}

Se considera también el factor y coeficiente de la relación de costos directos, para esto, debe recordarse lo indicado sobre el componente fundamental de todos los insumos que participan en un proceso productivo que es la materia prima, cuyo costo debe ser conceptualizado como referencia. Así, se define el factor que representa la influencia de la relación de los costos directos por personal, mano de obra, equipo y maquinaria con relación a los costos directos por materia prima y partes por lo que el factor y coeficiente son:

$f_{\text {rai }} \quad$ Factor de relación de los costos directos de mano de obra, equipo y maquinaria a los de materia prima del i-ésimo producto.

$\pi_{r c, i} \quad$ Coeficiente-Prorrata que relaciona, la relación de los costos de mano de obra, equipo y maquinaria a los de materia prima del i-ésimo producto al total.

Por otra parte, la relación de costos directos muestra cuán grande o pequeña es en la producción, la participación del personal, mano de obra, equipo y maquinaria, con relación a la materia prima. En realidad, lo que constituye al bien producido es la materia prima; sea un bien de consumo o de otro tipo.

Así, por ejemplo, mientras el costo final este más asociado a la materia que constituye el bien, entonces, menos atribuible a este, será el costo humano indirecto por: administración o comercialización. 
Se puede tener información adicional cuando se define otro factor que representa la influencia de la relación de los costos directos por equipo y maquinaria con relación a los costos directos por personal y mano de obra. Este factor dará información sobre el grado de mecanización del i-ésimo proceso productivo $g m_{, i}$, calculando con este:

fgm $_{i}$ Factor de relación de los costos directos de equipo y maquinaria a los de materia prima del i-ésimo producto.

$\pi g_{m, i} \quad$ Coeficiente-Prorrata, que muestra la relación de los costos de equipo y maquinaria con los de materia prima del i-ésimo producto.

Los factores y coeficientes que a continuación se presentan se referirán, eventualmente, como aquellos de contenido propio, indicando con ello que, si bien algunos pueden usarse en un prorrateo, en este estudio, servirán únicamente por su significado y la información que de ellos se pueda obtener. No debe olvidarse que la complejidad $y$, por tanto, el esfuerzo que demanda introducir estas relaciones matemáticas en el tratamiento de costos se realiza una sola vez, ya que posteriormente, el sistema informático ofrecerá la información cuando sea requerida.

\section{Factor y coeficiente del tiempo de ciclo}

Sin considerar los pedidos especiales, el tiempo de ciclo representativo en el estudio de costos, es el que se promedia de los valores que corresponden a los m-ésimos valores de lote de producto promedio. El tiempo de ciclo promedio es el tiempo que tarda la materia prima e insumos en convertirse en producto con el nombre de $t c$, Este indicador es importante, pero debe ir correlacionado con la cantidad de lote de producto promedio en \$, o sea $Q_{\$ i}$ Ambos datos deben ser vistos de manera simultánea porque pudiera apreciarse equivocadamente, como muy eficiente la producción de un i-ésimo producto que tiene bajo $t c_{i, i}$, solo porque su $Q_{\$ i}$ es muy pequeño. Entonces, como se hizo en el manejo de costos, este tiempo que es el que corresponde a un lote de producto promedio, se lo referirá a la unidad monetaria $\$ c$ lograda en la producción $t c_{\$, i}$

Cuando la producción se da con cierta uniformidad, tanto en número de lotes producidos al año, como en el tamaño de estos, esta consideración no es necesaria y todo puede ser tratado de forma más simple en unidades de lote.

El coeficiente prórrata $\pi_{t c, i}$ que no será utilizado en este estudio pero servirá de referencia al considerar el tiempo de recuperación de capital. También, se puede usar como un indicador de prorrateo que indique cómo, un proceso requiere más que otro, mayor tiempo de planta de mano de obra, de equipos y maquinaria. En el caso del Factor $f_{t c}$; de la relación de tiempos de ciclo esta muestra cuánto tarda en producirse un producto i-ésimo con relación a un producto hipotético que tiene el tiempo de ciclo promedio.

\section{Factor y coeficiente de salarios, operaciones, servicios y activos - SOSA}

Las variables de mayor peso a considerar son: a) tiempo de ciclo, b) tiempo que tarda en salir el producto de almacén de producto terminado $y, c)$ tiempo que éste permanece 
estacionado en puntos de venta. Los incisos a y b pueden considerarse para prorratear los costos indirectos de funcionamiento más significativos, como: salarios, operaciones, servicios y logística. Para todos estos cargos, se puede usar un único factor que represente el tiempo transcurrido entre comprar el insumo y lograr con la venta, una unidad monetaria \$, representado como $t_{T \$, i}$

Ahora, el coeficiente de prorrateo de los costos, que involucra: salarios, operación y servicios, se denomina $\pi_{S O S A}, i$ y es calculado con $t_{T \phi, i}$.Resumiendo lo tratado conceptualmente, se entenderá que todo producto que tiene un menor tiempo de ciclo y mayor velocidad de venta o, lo que es lo mismo, menor tiempo de salida de almacén, demanda menos costos indirectos.

\section{Factor y coeficiente de tiempo de venta}

Este coeficiente es muy similar al anterior y difiere únicamente en el hecho de que el tiempo considerado ahora, es el de venta (idéntico al de almacenaje) $t v_{\$ ; i}$ con el que se obtiene:

$f v_{, i} \quad$ Factor de relación del tiempo de venta de la unidad monetaria del i-ésimo producto, al tiempo promedio de los iésimos productos.

\section{$\pi t v,{ }_{i} \quad$ Coeficiente-Prorrata de la relación del tiempo de venta de la unidad monetaria del i-ésimo producto al total de los tiempos de venta de los i-ésimos productos.}

Según se observa, un producto que tiene un buen margen de utilidad al salir de planta, pero que tarda mucho en venderse, no gravitará considerablemente en el margen de utilidades. Por otro lado, tampoco repercutirá con resultados positivos, otro producto que se vende muy bien pero que no rinde utilidades.

Por tanto, de manera concluyente, se puede aseverar que no solo es importante vender más, sino también, más rápido y por supuesto, mientras exista margen de utilidad. Es importante analizar, que, dentro de las estrategias de comercialización, pudiera considerase como utilidad un beneficio colateral, aunque sea intangible pero cuyo impacto pueda ser estimado.

Una vez se han definido factores de ponderación y coeficientes de prorrateo, se procede a sintetizar el modelo general de costos.

\section{SÍNTESIS DE LOS COSTOS GLOBALES}

Se muestran primero los costos globales incluyendo los costos directos e indirectos considerando las bases de afectación y coeficientes de prorrateo, mostrando finalmente la síntesis del modelo completo.

\section{Costo global de materia prima y partes}

En el caso de materia prima y partes, se tiene el costo real, incluyendo mermas:

$$
\begin{gathered}
C_{M P P, i}=\Sigma_{j} q_{M P j}\left[1+\left(\mu_{M P j / 100}\right)\right] c_{M P, j}+\Sigma_{j} q \\
C P j\left[1+\left(\mu_{C P j / 100}\right)\right] c_{C P, j} \\
C_{M P P \$, i}=C_{M P P, i} / P_{L, i}
\end{gathered}
$$

El costo encontrado es aplicable en un proceso que considera una cantidad determinada y que ha sido aceptada en este estudio como la del lote de producto promedio y la normalizada como la de la unidad monetaria \$. 


\section{Costo global de personal y mano de obra}

El costo directo por Personal y Mano de Obra, es:

$$
C_{P M O, i}=\Sigma_{j\left[13 *\left(s_{p m o, j} \Delta t_{j)}\right]\right.} /(\text { Hrs-año })
$$

El costo global, incluyendo la parte de costo indirecto con su base y coeficiente es:

$C_{P M O, G, i}=C_{P M O, i}+\pi_{P M O, i} \cdot\left(\Delta_{P M O, i}\right) / N Q A_{i}$

\section{Costo global de instalaciones técnicas, equipo y maquinaria}

A continuación, se ve el costo directo (financiero y de depreciación) y el costo indirecto, incluyendo sus respectivas bases de afectación y coeficientes, formando así el costo global:

$$
\begin{aligned}
C_{I E M, G, i}= & C_{I E M, F, i}+\left\{\pi_{I E M, F, I}[C F \text { de IEM - }\right. \\
& \left.\left.\Sigma_{i} N Q A_{i} C_{I E M, F, I}\right]\right\} / N Q A_{i}
\end{aligned}
$$$$
+C_{I E M, D, i}+\left\{\pi _ { I E M , D , i } \left[C D \text { de IEM }-\Sigma_{i} N Q A_{i}\right.\right.
$$$$
\left.\left.C_{I E M, D, i}\right]\right\} / N Q A_{i}
$$

\section{Costo global por uso de almacén de producto terminado}

Se tiene asignado el total de los costos para el almacenamiento de producto, por lo que el costo global es:

$$
C_{A d Q i}=\left(C_{F A, m 2} A_{Q i, m 2}\right)
$$

Este costo se calcula en función del espacio que requiere un lote de producto promedio.

\section{Costo global por insumos directos complementarios}

El costo directo por insumos complementarios considerará únicamente él o los que significativamente forman parte del proceso productivo:

$$
C_{I N S, i}=C_{K W h, i}+C_{G A S, i}+C_{H 2 O, i}+
$$

Este costo se calcula en función del consumo medido para el lote de producto promedio.

\section{Costo global por bases afectables de funcionamiento-SOSA}

Las bases afectables por: carga salarial, gastos de operación $y$ servicios $y_{i}$ financiamiento y depreciación de activos, son:

$$
\begin{gathered}
B A_{, S A L}=S_{A D}+S_{C M}+S_{P G}+S_{O M} \\
B A_{, O y S}=G_{O y S} \\
B A_{, A C T}=A C T_{F}\left[\tau_{O P} /(100)\right]_{+} A C T_{, D}
\end{gathered}
$$

Usando el coeficiente de prorrateo SOSA:

$$
\pi_{S O S A, i}=\left[t_{T \$, i} /\left(\Sigma_{i}^{N} t_{T \$, i}\right)\right]
$$

Afectando la base encontrada, se tiene:

$$
\begin{gathered}
C_{S O S A, G i}=\pi_{S O S A, i} \cdot\left(B A_{, S A L}+B A_{, O y S}+B A_{, A C T}\right) \\
/ N Q A_{i}
\end{gathered}
$$

Como los datos de la base de afectación son anuales, se utiliza el número $N Q A_{i}$ de lotes de producto promedio ingresados en almacén para tener el dato por $Q_{L, i}$ La estima se hace más exacta a medida que se cuenta con información de varios años. 


\section{Costo global por bases afectables de estacionamiento de producto}

La base afectable por estacionamiento de producto es:

$$
B A, E S T=E S T_{, T O T}\left[\tau_{O P} /(100)\right]
$$

Este coeficiente, lleva la información de tiempo de venta del producto con relación a todos los productos y calculará el costo de estacionamiento.

$$
\pi t,_{i=} t v_{\$, i}, \Sigma_{i}^{N} t v_{\$, i}
$$

Afectando la base encontrada, se tiene:

$$
C_{E S T, G, i}=\left(B A, E S T * \pi t v,{ }_{i}\right) / N Q A_{i}
$$

Como los datos de la base de afectación son anuales, se utiliza el número $N Q A_{i}$ de lotes de producto promedio.

\section{Composición general del modelo de costos}

La aproximación que se realizará para que el sistema informático muestre los resultados finales, es el de "Efecto a Causa" tal como mostró en el árbol de correlación de variables (Figura 1), es decir, recorriendo el camino que va de la utilidad y costos totales, hacia los componentes o costos parciales.

Así, el costo total es:

$C_{T O T i}=C_{D I R i}+C_{I N D i}$

Siendo los costos directos.

$$
\begin{gathered}
C_{D I R i}=C_{M P P, i}+C_{P M O, i}+C_{I E M, i}+C_{A d Q, i}+ \\
C_{I N S, I}
\end{gathered}
$$

Los costos indirectos.

$$
\begin{gathered}
C_{I N D, i}=\left[C_{S A L, i}+C_{O y S, i}+C_{A C T, F D, i]}+C_{E S T, i}\right. \\
=C_{S O S A, G i}+C_{E S T, G, i}
\end{gathered}
$$

Usando los resultados encontrados recientemente en la síntesis de costos globales, el costo total por lote de producto promedio para el i-ésimo producto, resulta ser:

$$
\begin{aligned}
C_{T O T i}= & C_{M P P, i}+C_{P M O, G, i}+C_{I E M, G, i}+C_{A d Q i} \\
& +C_{I N S, i}+C_{S O S A, G, i}+C_{E S T, G, i}
\end{aligned}
$$

Una vez definida la síntesis de los costos globales, es factible aplicar el modelo para demostrar su impacto en los costos.

\section{APLICACIÓN DEL MODELO MATEMÁTICO}

A continuación, se presenta la información tras la aplicación del modelo matemático en el control de costos de una empresa productiva.

En el marco de esta investigación, el algoritmo a definir consiste en un conjunto cronológico de métodos, medios y procedimientos que conjuncionan adecuadamente, para lograr el objetivo. Así, para obtener un buen sistema informático, se requiere: (1) Recolección de la información primaria; (2) Ordenamiento, categorización y procesamiento de la información primaria sobre costos directos; (3) Concepción del procedimiento para el cálculo de las variables intermedias: factores y coeficientes; (4) Concepción del procedimiento para el cálculo de las variables dependientes de los costos globales; (5) Contar con un marco referencial de apoyo a lo indicado anteriormente, sobre la estructura de la base de datos, en cuanto a tablas, registros y simbología. 
Una vez cumplidos los cinco puntos anteriores, se procedió a obtener resultados, por un lado, los costos directos y, por otro lado, los indirectos. Para estos últimos se procedió primero a calcular las bases de afectación y coeficientes de prorrateo con los que finalmente se obtuvo los costos indirectos globales. La Tabla 1 muestra los resultados finales de siete productos escogidos para el análisis de este estudio (productos A, B, C, D, E, F y $G$ ).

\section{Tabla 1}

Desglose por tipo de costo de unidad monetaria

\section{Costos Directos y Costos Extendidos Globales de Producción por \$}

\begin{tabular}{|c|c|c|c|c|c|c|c|c|c|c|c|}
\hline & & & & & & & & & 10 & & \\
\hline & Código & $\overline{\text { NONBRE }}$ & $M P P \$, i$ & $\overline{C_{P M O S, G, i}}$ & $C_{I E M \&, G, i}$ & $C_{A d Q i}$ & $C_{I N S, i}$ & $C_{S A L}$ & $C_{\text {oys }}$ & $C_{A C T}$ & $\overline{C_{E S T, G, i}}$ \\
\hline & CAJRSA & PRODUCTO A & 0,318 & 197 & 0,044 & 0,000 & 0,004 & 0,050 & 0,079 & 0,023 & 0,028 \\
\hline & CAJBOM & PRODUCTO B & 0,224 & 27 & 238 & 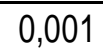 & 0,001 & 373 & 0,587 & 170 & 0,036 \\
\hline & CAJTRU & PRODUCTO C & 0,318 & 66 & 0,022 & 0,000 & 0,002 & 0,087 & 0,138 & 0,040 & 0,012 \\
\hline & TAB LEC & PRODUCTO D & 0,332 & 0,069 & 0,085 & 0,001 & 0,001 & 0,110 & 0,173 & 0,050 & 0,017 \\
\hline & TABAM & PRODUCTO E & 0,381 & 74 & 0,387 & 0,001 & 0,001 & 0,163 & 0,256 & 0,074 & 0,072 \\
\hline & CHOAMI & PRODUCTO F & 0,385 & 0,009 & 0,034 & 0,000 & 0,011 & 0,018 & 0,028 & 0,008 & 0,005 \\
\hline & CHOLEC & PRODUCTO G & 0,333 & 0,008 & 0,016 & 0,000 & 0,012 & 0,012 & 0,019 & 0,006 & 0,001 \\
\hline
\end{tabular}

La Tabla 1 refleja muy bien la relación de costos, porque es independiente del tamaño del lote de producto promedio. Aquí se puede adelantar que la contribución de los costos indirectos, adicionalmente de lo que lleva en costos directos, es elevada en el Producto $B$, lo cual lo pone en observación. Algo similar sucede con el producto $\mathrm{E}$.

También se ve que el costo de almacenamiento directo es muy bajo, a tal punto que se lo pudiera obviar en las estimas. Si bien el costo por $\mathrm{m}^{2}$ es bajo, el financiero por estacionamiento es elevado para los mismos productos, motivo por el que lo relativo a sus procesos, demanda y costos deben ser analizados para ver si es conveniente mantenerlos en anaqueles.

Es muy importante comprender que, el modelo se valida cuando se analizan las ventas en las que se destaca el producto $\mathrm{G}$ como el más vendido y el que intuitivamente, ya se suponía, llevaba menos costos indirectos, por su proceso en línea mecanizada de menor tiempo de ciclo y su mayor velocidad de venta. Es también de resaltar, que este producto tiene un bajo costo de mecanización, no obstante, el alto uso de maquinaria, sino porque el tamaño de lotes de producción es mucho mayor.

Si bien se podía intuir que los alto costos de $B$ y $E$ podrían ofrecer bajas utilidades, no se concluía en algo que ahora, por lo menos provisionalmente, se puede asegurar, y es que el producto $G$ subvenciona las pérdidas de los productos $B$ y $E$.

Según se observa, los costos indirectos por unidades monetarias $\$_{a}$ que más afectan son los salarios indirectos, las operaciones $y$ servicios, que indistintamente del tipo de producto, muestran porcentajes altos con relación al total. 
La tabla 1 permite realizar un análisis de costos comparativo por producto, por ejemplo, los productos $A, B$, y $E$ tienen un alto costo en personal y mano de obra con relación a los otros productos. También se observa que $B$ y $E$, a pesar de tener elevado costo de mano de obra, tienen elevado costo en equipo y maquinaria, por lo que es conveniente revisar sus procesos de producción. Adicionalmente $B$ y E tienen mayor costo de estacionamiento que se debe a la baja rotación de los productos respecto al resto.

\section{Tabla 2}

Desglose porcentual por tipo de costo de lote de producto promedio

\section{Porcentaje de Cada Tipo Costo por Producto}

\begin{tabular}{|c|c|c|c|c|c|c|c|c|c|c|c|}
\hline \multirow[b]{2}{*}{ Códig } & \multirow[b]{2}{*}{ NONBRE } & \multirow[b]{2}{*}{$C_{M P P, i}$} & \multirow[b]{2}{*}{$C_{P M O, G, i}$} & \multirow[b]{2}{*}{$C_{I E M, G, i}$} & \multirow[b]{2}{*}{$C_{A d Q i}$} & \multirow[b]{2}{*}{$C_{i N S, i}$} & \multicolumn{3}{|c|}{$C_{\text {SOSA,Ti }}$} & \multirow[b]{2}{*}{$C_{E S T, G, i}$} & \multirow[b]{2}{*}{$\begin{array}{c}\text { TOTAL } \\
\%\end{array}$} \\
\hline & & & & & & & $\tilde{C}_{S A L}$ & $C_{\text {OyS }}$ & $C_{A C T}$ & & \\
\hline CAJRSA & PRODUCTO A & 43 & 27 & 6,0 & 0,04 & 0,5 & 7 & 11 & 3 & 4 & 100 \\
\hline CAJBOM & PRODUCTO B & 11 & 21 & 11,6 & 0,07 & 0,0 & 18 & 29 & 8 & 2 & 100 \\
\hline \begin{tabular}{|l|} 
CAJTRU \\
\end{tabular} & PRODUCTO C & 41 & 21 & 2,8 & 0,03 & 0,3 & 11 & 18 & 5 & 2 & 100 \\
\hline \begin{tabular}{|l|} 
TAB LEC \\
\end{tabular} & PRODUCTO D & 40 & 8 & 10,2 & 0,08 & 0,1 & $\sqrt{3}$ & 21 & 6 & 2 & 100 \\
\hline TABAM & PRODUCTO E & 25 & 12 & 25,6 & 0,08 & 0,1 & 11 & 17 & 5 & 5 & 100 \\
\hline CHOAMI & PRODUCTO $\mathrm{F}$ & 77 & 2 & 6,7 & 0,04 & 2,1 & 4 & 6 & 2 & 1 & 100 \\
\hline CHOLEC & PRODUCTO G & 82 & 2 & 4,1 & 0,05 & 2,8 & 3 & 5 & 1 & 0 & 100 \\
\hline
\end{tabular}

En la Tabla 2 se aprecia, cómo influye porcentualmente cada tipo de costo respecto al total, observándose la gran incidencia que tienen los costos indirectos SOSA. Se destaca también algo importante sobre los productos $B$ y $E$ que muestran en general, altos costos de maquinaria y mano de obra. Adicionalmente, se observa que, con relación a la materia prima, en el primero, predomina la mano de obra mientras que, en el segundo, predomina el uso de equipo y maquinaria.

Ahora, en la Tabla 3, se presentan los resultados finales, en $\%$ y en relación de unidades monetarias.

En la Tabla 3, se puede observar la influencia de los costos indirectos que, en algunos casos, contrariamente a lo que se busca en un sistema productivo, resultan ser más altos que los directos.

Como se adelantó, las estimas muestran que los dos productos $B$ y $E$, dan pérdida en vez de utilidad con una razón de costo en unidades monetarias $\$_{a}$ a unidades monetarias recibidas en la venta $\$_{v}$ mayor a la unidad. Concretamente, para recibir una unidad monetaria al vender el producto $B$, por ejemplo, se ve que se debe gastar más de 2 unidades monetarias, confirmando su permanencia en el espectro de ofertas al mercado, está subvencionada por otros productos. 


\section{Tabla 3}

Presentación de los costos finales y costo por unidad monetaria

\begin{tabular}{l}
\multicolumn{3}{|l}{ Utilidades Totales } \\
\begin{tabular}{|c|l|ll|}
\hline $\mathrm{i}$ & Código & NONBRE \\
\hline 1 & CAJRSA & PRODUCTO & A \\
\hline 2 & CAJBOM & PRODUCTO & B \\
\hline 3 & CAJTRU & PRODUCTO & C \\
\hline 4 & TAB LEC & PRODUCTO & D \\
\hline 5 & TABAM & PRODUCTO & E \\
\hline 6 & CHOAMI & PRODUCTO & F \\
\hline 7 & CHOLEC & PRODUCTO & G \\
\hline
\end{tabular}
\end{tabular}

\begin{tabular}{|c|c|c|c|c|c|c|}
\hline \multicolumn{3}{|c|}{ Unitarios } & & & & \\
\hline Directo & Indirecto & Total & & & & \\
\hline$C_{D I R}$ & $C_{I N D}$ & $C_{T}$ & $\begin{array}{l}\text { COSTO DE LA } \\
\text { EMPRESA (Bs) }\end{array}$ & $\begin{array}{c}\text { PRECIO (Bs) } \\
\text { Sin IVA -IT }\end{array}$ & $\begin{array}{l}\text { UTILIDAD } 0 \\
\text { PERDIDA \% }\end{array}$ & $C i \$ p \$$ \\
\hline 17 & 11 & 28 & 22 & 38 & 26 & 0,74 \\
\hline 11 & 37 & 47 & 16 & 23 & $(-106)$ & 2,06 \\
\hline 19 & 13 & 32 & 25 & 41 & 21 & $\overline{0,79}$ \\
\hline 6 & 7 & 13 & 8 & 16 & 16 & 0,84 \\
\hline 8 & 20 & 28 & 13 & 19 & $-51)$ & 1,51 \\
\hline 46 & 10 & 57 & 50 & 114 & 50 & 0,50 \\
\hline 38 & 5 & 43 & 38 & 106 & 59 & 0,41 \\
\hline
\end{tabular}

Se reafirma entonces, la necesidad de revisar para estos productos: procesos, costos inmersos, grado de estacionamiento, nivel de ventas y precios de la competencia, si la hubiere.

Realizando un análisis general de los resultados, se observa que la simulación, confirmó en alto grado, la capacidad del modelo para ofrecer información sobre algunos hechos que están muy lejos de predecirse sin su aplicación, entre ellos que:

- Los productos A, C y D tienen utilidades, pero bajas que se puede mejorar en caso de aumentar la producción, por lo que es aconsejable impulsarlos siempre y cuando la empresa pueda incrementar su capacidad de producción.

- Lo más destacable, es lo encontrado sobre los productos B y E que según se aprecia, generan gran pérdida. $B$ es de alta demanda y uno de los productos estrella, por lo que sería recomendable automatizar el proceso y reducir costos indirectos. Si esto no es posible, este debe ser dado de baja cuanto antes. Algo importante también es que el precio de $B$ es relativamente bajo en comparación con los de la competencia y a productos similares por lo que es posible realizar un ajuste.

- Los productos D y E son similares, sin embargo, D tiene mayor demanda con una producción masiva que hace que sus costos sean más bajos que los de E. E también tiene un costo más alto porque contiene mayor concentración de la materia prima de mayor precio.

- Los costos unitarios calculados con el modelo son, naturalmente, mayores que los usados actualmente por la empresa, porque el modelo incluyó los costos indirectos e información adicional que incide significativamente en los resultados finales.

Para concluir, debe tenerse presente que, del total de productos considerados en la simulación, solamente 20 fueron elegidos para análisis detallado y de estos, a su vez, solamente 7 se expusieron, ya que se diferenciaban en tipo de procesos y contenidos de materia prima, a fin de validar el modelo e ilustrar su aplicabilidad y efectividad. 


\section{CONCLUSIONES}

Se observa de manera concluyente que el modelo permite identificar los costos que reducen las utilidades, entregando información valiosa a la empresa para una adecuada administración. Asimismo, se entiende que el problema también está presente en empresas del exterior y que, por tanto, el modelo puede ser de utilidad en otros países, sin tener que limitar su perspectiva de aplicación, solamente a Bolivia. No obstante, es importante tomar en cuenta que, para la adecuada aplicación del modelo, se debe analizar el tipo de empresa, el sector empresarial al que pertenece y el país en el que se empleará, a fin de realizar su apropiación y ajustes necesarios. Complementariamente, se ha visto que un ámbito propicio para la toma de decisiones es posible solamente si se tiene la información adecuada y una base de datos correctamente estructurada.

Considerando que el modelo fue desarrollado con el marco de referencia para informatizarlo, la inversión requerida para su programación e implantación es mínima, por lo que es recomendable analizar su implantación en toda empresa que tenga dudas sobre la eficiencia con la que se manejan sus costos indirectos.

La información ofrecida por el modelo puede lograr disminuir el tiempo improductivo del personal de planta, optimizar la maquinaria, equipo e instalaciones de producción, disminuir y mejorar el área útil de almacenaje, reducir el personal administrativo en general, optimizar el mantenimiento del stock, reducir el estacionamiento de producto y disminuir los saldos deudores de los clientes, entre otros.

Los resultados de la simulación muestran algunas novedades desde que se hacen las primeras estimas. Al concluir, en la información final, se ve que existen productos con buena y baja utilidad, y se pueden confirmar las apreciaciones intuitivas existentes sobre productos que dan pérdida y que ahora al resultar con valores negativos de utilidad, validan el modelo. Existen beneficios materiales y cualitativos que resultan de su aplicación, por lo que toda empresa que pretenda obtener mayores utilidades, optimizando sus recursos humanos y materiales, puede beneficiarse de la implantación del modelo.

\section{REFERENCIAS}

Castán, J. M.; Pisa, C. C. \& Carballosa, A. N. (2003). La logística en la empresa: fundamentos y tecnologías de la información y de la comunicación. Ediciones Pirámide

Christopher, M.; Sharman, G. \& Langley J. (2010). Logística: Aspectos estratégicos. México: Limusa

Hansen, D. R.; Mowen, M. M.; Quiñones, A. D. \& Fragoso, F. S. (2007). Administración de costos: contabilidad y control. International Thomson

Hernández, S R.; Fernández, C. \& Baptista M.P. (2010). Metodología de la Investigación 3ra ed. México: Mcgraw-hill / Interamericana Editores

Neuner, J. \& Deakin, E. (2005). Contabilidad de costos: Principios y prácticas. Editorial Limusa, $S A$

Montoya, L. A.; De Arias, L. M. P. \& Henao, S. A. F. (2010). La distribución de costos indirectos de fabricación, factor clave al costear productos. Scientia et technica

Rincón-Soto, C. A.; Sánchez-Mayorga, X. \& Cardona-Restrepo, L. M. (2019). Clasificación teórica de los costos. Revista EAN, (87), 193-206

Sánchez B. B. J. (2009). Problemática de conceptos de costos y clasificación de costos 
William, J. L. (2010). Contabilidad de costos. Bogotá DC Colombia. Editorial Fundación 